\title{
Cancer-associated thrombosis: the when, how and why
}

\author{
Caio J. Fernandes (D, Luciana T. K. Morinaga, José L. Alves Jr, \\ Marcela A. Castro, Daniela Calderaro, Carlos V. P. Jardim and Rogerio Souza
}

Affiliation: Dept of Cardiopulmonology, Heart Institute, University of Sao Paulo Medical School, Sao Paulo, Brazil.

Correspondence: Caio J. Fernandes, Dept of Cardiopulmonology, Heart Institute, University of Sao Paulo Medical School, 44, Av. Dr. Eneas de Carvalho Aguiar, Sao Paulo, 05403-000, Brazil. E-mail: cjcfernandesla yahoo.com.br

@ERSpublications

Cancer-associated thrombosis (CAT) presents peculiar features (risk factors and pathophysiology) that distinguish it from common VTE cases. Treatment of CAT requires a different approach, since the patients are more prone to recurrence and bleeding. http://ow.ly/j1Lu30nYmd5

Cite this article as: Fernandes CJ, Morinaga LTK, Alves JL Jr, et al. Cancer-associated thrombosis: the when, how and why. Eur Respir Rev 2019; 28: 180119 [https://doi.org/10.1183/16000617.0119-2018].

ABSTRACT Cancer-associated thrombosis (CAT) is a condition in which relevance has been increasingly recognised both for physicians that deal with venous thromboembolism (VTE) and for oncologists. It is currently estimated that the annual incidence of VTE in patients with cancer is $0.5 \%$ compared to $0.1 \%$ in the general population. Active cancer accounts for $20 \%$ of the overall incidence of VTE. Of note, VTE is the second most prevalent cause of death in cancer, second only to the progression of the disease, and cancer is the most prevalent cause of deaths in VTE patients. Nevertheless, CAT presents several peculiarities that distinguish it from other VTE, both in pathophysiology mechanisms, risk factors and especially in treatment, which need to be considered. CAT data will be reviewed in this review.

\section{Introduction}

Venous thromboembolism (VTE) is a highly prevalent and potentially fatal disease. It is the third most common cause of cardiovascular death, following acute coronary artery disease and stroke, and is responsible for more than 3 million deaths per year worldwide [1,2]. Several risk factors have been associated with VTE such as obesity, hormone use and immobility among others, but none as relevant as cancer. It is currently estimated that the annual incidence of VTE in patients with cancer is $0.5 \%$ compared to $0.1 \%$ in the general population [3]. Active cancer accounts for $20 \%$ of the overall incidence of VTE $[4,5]$. More important than that, cancer is a major cause of death in VTE patients and vice versa. Data from the Framingham Heart Study demonstrated that in a prospective cohort of 9754 patients, cancer-associated thrombosis (CAT) had worse survival among VTE patients [6]. In the same direction, data from the Global Anticoagulant Registry in the Field (GARFIELD)-VTE registry demonstrated that in a cohort of 10315 VTE patients, from 419 centres and 28 countries, overall mortality was $9.7 \%$ in 6 months and $54.3 \%$ of all deaths were cancer related [7]. VTE is the second most prevalent cause of death from cancer, second only to cancer itself [8].

CAT presents several peculiarities that distinguish it from other VTE and are the target of intense interest in recent medical literature. Abundant data have been generated concerning CAT and its distinct risk factors, pathophysiological mechanisms, difficulties in the diagnosis and specificities concerning treatment, and will be addressed in this review.

Received: Dec 192018 | Accepted after revision: March 012019

Provenance: Submitted article, peer reviewed.

Copyright CERS 2019. This article is open access and distributed under the terms of the Creative Commons Attribution Non-Commercial Licence 4.0. 


\section{Pathophysiological mechanisms of CAT}

The thrombotic generation process in the cancer patient is distinct from the non-cancer population. A protein that is considered critical to CAT is tissue factor, which plays a role both in oncologic progression and in VTE formation. It is abnormally produced by cancer cells, and plays the role of an activator of the extrinsic coagulation pathway resulting in the activation of factor $\mathrm{X}$ and consequently in fibrin synthesis and platelet activation. Besides tissue factor, some cancer cells can also produce other substances, such as distinct cancer pro-coagulant factors that directly stimulate factor Xa, inflammatory cytokines that mediate endothelial dysfunction and other tumoral produced substances, such as carcinoma mucins, that also interfere in the coagulation cascade. The fibrinolytic system is also inhibited by the cancer cell synthesised plasminogen activator inhibitor-1. This imbalance in the pro-anticoagulation balance leads to CAT generation and its clinical repercussions [9].

\section{CAT risk factors}

In 1856, Rudolf Virchow postulated a triad of conditions that lead to thrombosis: endothelial injury, circulatory stasis, and abnormalities in blood clotting components (hypercoagulable state) [10]. Cancer patients have several conditions that predispose them to thrombus generation. Their treatment often requires multiple surgical procedures, with the placement of long-term catheters and chemotherapy (endothelial injury). Cancer may also contribute to the development of circulatory stasis since bulky tumoral vascular compression may occur, reducing the blood flow locally. Oncologic pain also can cause immobility, further worsening damaged venous drainage. Since the late 19th century, it has been known that some kinds of cancer are associated with an increased blood viscosity and acquired thrombophilia [11]. More recently, tumour genetic characteristics also appear to play a role in VTE formation, since genetic profiling demonstrated that mutations in K-ras in colon and lung cancer show an association with increased risk of VTE [12, 13]. JAK2 V617F, a mutation frequently present in patients with myeloproliferative cancer [14], is also associated with an increased risk of VTE [15].

Different cancer types carry different VTE risk. Haematological malignancies, lung, pancreas, stomach, bowel and brain cancers are associated with a high risk of clot formation $[16,17]$, whilst prostate and breast cancers are associated with low risk of thrombosis [18]. Nevertheless, despite the low relative risk, since prostate and breast are two of the most prevalent cancers worldwide, VTE is commonly seen in these populations. The risk of VTE in patients with a diagnosis of malignancies within 5 years is shown in table 1 [19].

Other risk factors have been described as contributing to CAT [20]: higher tumour grade, metastatic disease, surgery, haematopoiesis stimulating agents and chemotherapy, mainly platinum derivates, taxane-based agents, tyrosine kinase inhibitors and immunomodulatory drugs. Particularly worrisome is the use of angiogenesis inhibitors since they disturb endothelial homeostasis and are prone to VTE generation [21]. Main risk factors for CAT are summarised in table 2.

In an attempt to foresee VTE events in cancer patients, risk scores pondering the factors previously described have been created. Perhaps the one most used is the Khorana score (table 3) [24]. It confers points according to patient and tumour characteristics and is able to classify CAT risk into three categories: high risk (7\% VTE events in the subsequent 2.5 months), intermediate risk $(2 \%)$ and low risk $(\sim 0.5 \%)$.

TABLE 1 Relative risk of venous thromboembolism diagnosis in hospitalised cancer patients

Cancers

Odds ratio

Haematological cancer
Lung
Gastrointestinal (bowel, pancreas, stomach, oesophagus)
Brain
Kidney
Skin (melanoma, squamous cell)
Breast
Prostate
Uterine cervix
Ovarium
Ear, nose and throat
Other

Data from [19]. 


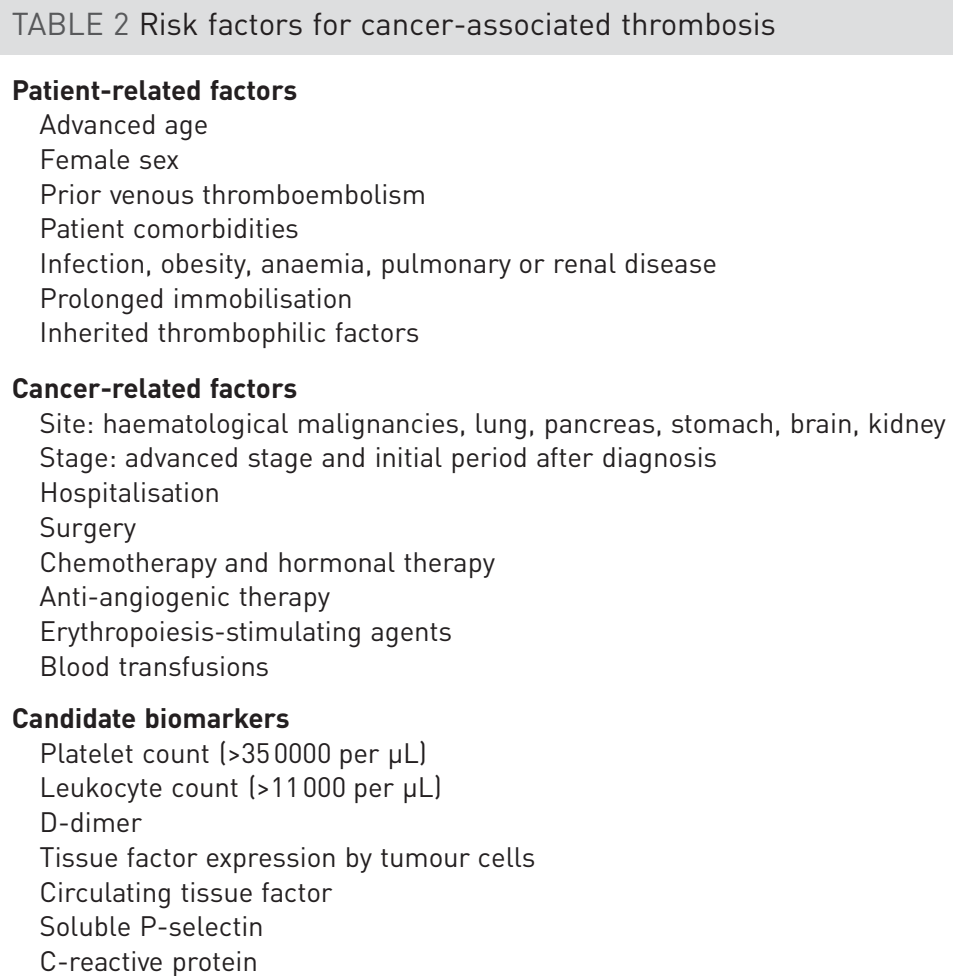

\section{Cancer-related factors}

Site: haematological malignancies, lung, pancreas, stomach, brain, kidney

Stage: advanced stage and initial period after diagnosis

Hospitalisation

Surgery

Chemotherapy and hormonal therapy

Anti-angiogenic therapy

Erythropoiesis-stimulating agents

Blood transfusions

\section{Candidate biomarkers}

Platelet count ( $>350000$ per $\mu \mathrm{L}$ )

Leukocyte count ( $>11000$ per $\mu \mathrm{L}$ )

D-dimer

Tissue factor expression by tumour cells

Circulating tissue factor

Soluble P-selectin

$\mathrm{C}$-reactive protein

Reproduced from [22] with permission from the publisher.

Another validated CAT risk score in use is the Vienna score [25]. Risk scores allow for: patient education; increasing VTE awareness and its potential morbidity in cancer patients; VTE screening in high-risk, asymptomatic cancer patients; and, in selected cases, for the use of primary VTE prophylaxis in cancer outpatients [26].

\section{CAT primary prophylaxis}

Since CAT carries a high VTE recurrence risk, as well as a high risk of major bleeding with anticoagulation, it makes sense to evaluate individual VTE risk in cancer patients and, in high-risk patients, promote low dosage anticoagulation as primary prophylaxis. The Khorana score (table 3) and Vienna score, among others, can be used for risk stratification of VTE possibility based on clinical history and basic blood tests.

Several pharmacological agents have been studied for primary prophylaxis of CAT in different scenarios. In 2009, the PROTECHT (Prophylaxis of Thromboembolism during Chemotherapy) study evaluated ambulatory patients receiving chemotherapy for metastatic or locally advanced solid cancer (lung, gastrointestinal, pancreatic, breast, ovarian, head, and neck) [27]. 1150 patients were randomised 2:1 to receive nadroparin, a low molecular-weight heparin (LMWH) (3800 U once daily), or placebo, for up to

\section{TABLE 3 Predictive model for chemotherapy-associated venous thromboembolism}

\section{Patient characteristics}

Risk score

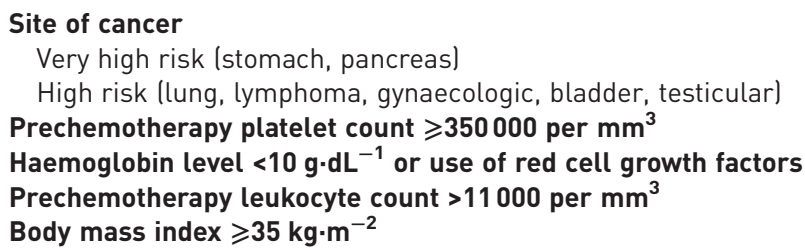

A risk score $\geqslant 3$ is a high-risk score, $1-2$ is an intermediate score, and 0 is a low-risk score. 
4 months. $2 \%$ of the nadroparin group presented with thromboembolic events (venous and arterial) versus $3.9 \%$ in the placebo group $(\mathrm{p}=0.02)$. About $0.7 \%$ of the nadroparin group presented with major bleeding versus none in the placebo group $(\mathrm{p}=0.18)$. However, the thrombosis event rate in the untreated population was lower than expected, and despite the relative risk reduction of $50 \%$, the absolute reduction of the thrombosis rate in PROTECHT with the LMWH was low (15 out of 767 patients, 52.6\% patients needed to receive nadroparin to avoid one thrombotic event).

In 2012, the SAVE-ONCO (Semuloparin for Thromboprophylaxis in Patients Receiving Chemotherapy for Cancer) study evaluated the benefit of semuloparin, an ultra LMWH, in this context [28]. A total of 3212 patients with metastatic or locally advanced solid tumours who started chemotherapy were randomly assigned to semuloparin $20 \mathrm{mg}$ once daily or placebo, until a change in the chemotherapy regimen. Median treatment duration was 3.5 months. VTE occurred in $1.2 \%$ of the semuloparin group versus $3.4 \%$ of the placebo (HR 0.36, 95\% CI 0.21-0.60; p<0.001), with consistent efficacy among subgroups defined according to the origin and cancer stage. No difference was found in the major bleeding rates (2.8\% versus 2\%; HR 1.4, 95\% CI 0.89-2.21). Nevertheless, despite the exciting results from the SAVE-ONCO study, semuloparin is not commercially available. Recently, a meta-analysis evaluated data about primary prophylaxis in CAT and concluded that primary prophylaxis with LMWH significantly reduced the incidence of symptomatic VTE in ambulatory cancer patients treated with chemotherapy [29]. The risk of major bleeding associated with LMWH suggested caution and required additional studies to determine the risk-to-benefit ratio of LMWH. Therefore, routine prophylaxis in ambulatory cancer patients with LMWH cannot be recommended before safety issues are adequately addressed.

With the advent of direct oral anticoagulants for VTE treatment and their favourable pharmacological profile, this family of drugs has also been considered for CAT primary prophylaxis. In the AVERT (Apixaban for the prevention of Venous Thromboembolism in High Risk Ambulatory Cancer Patients) study [30], 563 ambulatory patients with cancer who were at intermediate-to-high risk for venous thromboembolism (Khorana score $\geqslant 2$ ) and starting chemotherapy were randomised to receive apixaban $2.5 \mathrm{mg}$ twice daily or placebo for 180 days. Venous thromboembolism occurred in 12 (4.2\%) out of 288 patients in the apixaban group and in $28(10.2 \%)$ out of 275 patients in the placebo group (HR $0.41,95 \%$ CI $0.26-0.65 ; \mathrm{p}<0.001)$. Major bleeding occurred in 10 (3.5\%) patients in the apixaban group and in five $(1.8 \%)$ patients in the placebo group (HR 2.00, 95\% CI 1.01-3.95; $\mathrm{p}=0.046$ ). In the same direction, the CASSINI (Rivaroxaban for Preventing Venous Thromboembolism in High-Risk Ambulatory Patients with Cancer) trial evaluated 841 patients starting a new systemic cancer treatment and at ancreased risk of developing VTE (Khorana score $\geqslant 2$ ) who were randomised to receive rivaroxaban $10 \mathrm{mg}$ once daily or placebo for up to 180 days [31]. Subjects were screened with lower extremity ultrasounds every 8 weeks during the study to look for asymptomatic deep vein thrombosis. Primary efficacy end-point was a composite of objectively confirmed symptomatic or asymptomatic VTE and VTE-related death. It occurred in 25 (5.95\%) out of 420 patients and $37(8.79 \%)$ out of 421 patients (HR 0.66, 95\% CI $0.40-1.09 ; \mathrm{p}=0.101$ ) in the rivaroxaban and placebo groups (number needed to treat=35), respectively. Major bleeding occurred in eight $(1.98 \%)$ out of 405 in the rivaroxaban group and in four (0.99\%) out of 404 patients in the placebo group (HR 1.96, 95\% CI 0.59-6.49; $\mathrm{p}=0.265$ ) (number needed to harm=101). All-cause mortality occurred in $20.0 \%$ of patients in the rivaroxaban group and $23.8 \%$ in the placebo group $(\mathrm{HR}=0.83,95 \% \mathrm{CI} 0.62-1.11 ; \mathrm{p}=0.213)$. A pre-specified composite of the primary end-point with all-cause mortality occurred in $23.1 \%$ of patients in the rivaroxaban group and $29.5 \%$ in the placebo group (HR $0.75,95 \%$ CI $0.57-0.97 ; p=0.03$ ). However, only $62.4 \%$ completed the whole period of the study; withdrawal of consent and death were the primary reasons for discontinuation. Even so, data from these two studies suggest that direct oral anticoagulants may play a role in CAT primary prophylaxis in high-risk VTE patients (defined as Khorana score $\geqslant 2$ ).

While primary prophylaxis in ambulatory CAT is still controversial, in surgical cancer patients, particularly the ones submitted to undergo major abdominal procedures, there is a clear definition for the LMWH prophylactic use [32]. The ENOXACAN (Efficacy and safety of enoxaparin versus unfractionated heparin for prevention of deep vein thrombosis in elective cancer surgery: a double-blind randomised multicentre trial with venographic assessment) II study evaluated 332 cancer patients admitted for abdominal or pelvic surgery and randomised them to receive enoxaparin $40 \mathrm{mg}$ once daily for 31 days or placebo after the initial 10 days. The enoxaparin group presented with less VTE than placebo at 1 month ( $4.8 \%$ versus $12 \%, \mathrm{p}=0.02$ ) and 3 months $(5.5 \%$ versus $13.8 \%, \mathrm{p}=0.01)$, without any difference in the bleeding rates. These results led to the recommendation for LMWH use as primary prophylaxis in all cancer patients submitted to major abdominal/pelvic surgical procedures, for at least 30 days (table 4) [33].

\section{Treatment of CAT}

Overall, CAT therapy follows the same principles of VTE treatment, but some remarks should be emphasised. Efficacy and safety of anticoagulation are not the same between cancer and non-cancer 
TABLE 4 Major randomised trials in cancer-associated thrombosis primary prophylaxis

\begin{tabular}{|c|c|c|c|c|}
\hline Trial & Year & Patients n & Drugs evaluated & Major findings \\
\hline $\begin{array}{l}\text { PROTECHT } \\
\text { [27] }\end{array}$ & 2009 & 1150 & $\begin{array}{l}\text { Nadroparin ( } 4 \text { months) versus } \\
\text { placebo }\end{array}$ & $\begin{array}{c}\text { Nadroparin patients presented less thrombotic events } \\
\text { (venous and arterial) than placebo ( } p=0.02 \text { ). No statistical } \\
\text { difference in major bleeding. }\end{array}$ \\
\hline AVERT [30] & 2018 & 563 & $\begin{array}{c}\text { Apixaban (180 days) versus } \\
\text { placebo }\end{array}$ & $\begin{array}{c}\text { Apixaban patients presented less VTE events than placebo } \\
(p<0.001) \text { and more major bleeding }(p=0.046) \text { than } \\
\text { placebo. }\end{array}$ \\
\hline CASSINI [31] & 2018 & 841 & $\begin{array}{c}\text { Rivaroxaban (180 days) versus } \\
\text { placebo }\end{array}$ & $\begin{array}{l}\text { Statistical trend of less VTE events and VTE-related death } \\
\text { in rivaroxaban patients }(p=0.10) \text {. No difference in major } \\
\text { bleeding. }\end{array}$ \\
\hline
\end{tabular}

VTE: venous thromboembolism.

patients. In a now classic Italian prospective cohort of 842 VTE patients treated with vitamin $\mathrm{K}$ antagonist (VKA), that included 181 patients with CAT, thrombosis recurrence in 12 months was $20.7 \%$ (95\% CI 15.6-25.8\%) in the cancer population and 6.8\% (95\% CI 3.9-9.7\%) in the patients without cancer. Bleeding also proved to be an issue in CAT therapy: while major bleeding occurred in $12.4 \%$ (95\% CI 6.5 $18.2 \%$ ) in the cancer population, it only occurred in $4.9 \%$ (95\% CI $1.2-4.1 \%$ ) in patients without cancer [34]. Risk of VTE recurrence and bleeding correlated to the extension of cancer. This illustrates that in a fragile population such as cancer patients, despite treatment, risks of VTE are higher; however, they are more prone to the most severe adverse event of anticoagulant therapy, bleeding. This led to the pursuit of several anticoagulant strategies, searching for better and safer options for CAT treatment.

Long-term use of LMWH

Until 2002, long-term anticoagulation for CAT treatment was primarily by means of oral VKA. Nevertheless, VKA pharmacological properties impose serious difficulties to cancer patients. Constant drug interactions, malnutrition, vomiting, kidney and liver dysfunction and chemotherapy-induced thrombocytopenia, which are so frequent in this population, could lead to unpredictable anticoagulation levels and be harmful to the patients. LMWH was already used for CAT treatment, but mainly during the first week after VTE diagnosis, and then replaced by VKA. It was a natural evolution in an attempt to pursue a more stable anticoagulant effect, to extend the LMWH period of use to long term in CAT.

The CANTHANOX (Comparison of low-molecular-weight heparin and warfarin for the secondary prevention of venous thromboembolism in patients with cancer) trial was the first to evaluate this approach in a systematic way [35]. In 2002, in an open-label trial, it evaluated 146 CAT patients from 25 centres randomised to subcutaneous enoxaparin $\left(1.5 \mathrm{mg} \cdot \mathrm{kg}^{-1}\right.$ once daily) or warfarin, international normalised ratio (INR) guided dosage, for at least 3 months. Warfarin patients presented with more events in the predefined composite outcome (major bleeding or recurrent VTE), 15 (21.1\%, 95\% CI 12.3-32.4\%) compared with seven $(10.5 \%$ - 95\% CI, 4.3-20.3\%; $\mathrm{p}=0.09)$ of the patients assigned to receive enoxaparin. There were six deaths due to haemorrhage in the warfarin group compared with none in the enoxaparin group. In the warfarin group, 17 (22.7\%) patients died (95\% CI 13.8-33.8\%) compared with eight (11.3\%, $95 \%$ CI $5-21 \%$; $\mathrm{p}=0.07)$ in the enoxaparin group. No difference was observed regarding the progression of underlying cancer (weakening the hypothesis then present that heparin would interfere in the metastasis process and reduce cancer dissemination) or cancer-related death.

The study that went on to guide CAT therapy for the following 15 years was published in the following year in 2003. In the CLOT (Randomised Comparison of Low-Molecular-Weight Heparin versus Oral Anticoagulant Therapy for the Prevention of Recurrent Venous Thromboembolism in Patients with Cancer) trial, 672 patients from 48 centres in eight countries were randomised to receive dalteparin at a dose of 200 IU per kg of body weight subcutaneously once daily for 5-7 days and a VKA for 6 months, INR-guided dosage, or dalteparin alone for 6 months (200 IU per kg once daily for 1 month, followed by a daily dose of $150 \mathrm{IU}$ per $\mathrm{kg}$ for 5 months) [36]. Compared to the VKA group, dalteparin-treated patients had fewer recurrent VTE events ( $9 \%$ versus 17\%, $\mathrm{p}=0.002$ ). No significant difference between the dalteparin group and the oral anticoagulant group was detected in the rate of major bleeding ( $6 \%$ and $4 \%$, 
respectively) or overall mortality (39\% in the dalteparin group versus $41 \%$ in the VKA group). The CLOT trial demonstrated the difficulty of achieving good quality anticoagulation with VKA in cancer patients. In this study, the time in therapeutic range (the percentage of INR that remains in the target zone, among 2 and 3) of the VKA group was only $46 \%$, while the recommendation for effective anticoagulation is $>60 \%$. The CLOT results elicited a recommendation of LMWH as first-choice therapy for CAT treatment, which is still present in most VTE guidelines [33].

The CLOT results were somehow reproduced in another large trial, 12 years later. In the CATCH (Comparison of Acute Treatments in Cancer Hemostasis) study, 900 CAT patients from 164 centres were randomised to receive either tinzaparin $175 \mathrm{U} \cdot \mathrm{kg}^{-1}$ or warfarin, INR guided dosage, for 6 months [37]. Again, the anticoagulation quality achieved with VKA was low, with a time in therapeutic range of just $47 \%$. Recurrent VTE was reduced in the tinzaparin group (7.2\% versus $10.5 \%)$, but this time the difference did not achieve statistical significance $(\mathrm{p}=0.07)$. No difference was identified in major bleeding or overall survival. In the CATCH study, VTE recurrence was lower than expected, based on the previous CLOT data, probably due to the population included: less metastatic cancer, less chemotherapy use and more Asian patients, who are known to have a lower risk of thrombosis than Europeans. However, the CATCH trial was relevant due to its large sample size, and results that pointed in the same direction of CLOT. More recently, two meta-analyses re-evaluated the available data in LMWH and VTE treatment in cancer and ratified the role of LMWH in CAT, both in the initial anticoagulation period [38] and in the long term [39].

Nevertheless, despite guideline recommendations, the use of LMWH for CAT treatment is not easily done. Low patient adherence, local pain due to the method of administration and costs are some of the problems encountered. A study that demonstrates the difficulty of LMWH in CAT is the DALTECAN (Treatment of venous thromboembolism in cancer patients with dalteparin for up to 12 months) study [40]. In DALTECAN, an observational study of 334 CAT patients treated with dalteparin $200 \mathrm{IU}$ per kg once daily for 1 month, followed by a daily dose of $150 \mathrm{IU} \mathrm{kg}$ for 11 months, $10.2 \%$ of CAT patients presented with at least one episode of major bleeding and $11.1 \%$ presented with VTE recurrence in 1 year. Long-term adherence to subcutaneous treatment was low, with only $33 \%$ of patients receiving LMWH until the end of the study. In other words, despite significant advances in CAT treatment with LMWH, current guideline recommendations of CAT treatment still associate a strategy of a high risk of recurrence and bleeding with low patient adherence.

\section{Direct oral anticoagulants}

In 2009, direct oral anticoagulants were introduced for VTE treatment. These new drugs, one direct thrombin inhibitor (dabigatran) and three Xa antagonists (rivaroxaban, apixaban and edoxaban) proved to be, in large randomised controlled trials, at least as effective as the conventional VKA anticoagulant strategy for prevention of VTE recurrence in a general population. Their great advantage, however, was in safety; bleeding rates were significantly reduced with direct oral anticoagulant use [41]. These data led to the indication of direct oral anticoagulants as the first-choice anticoagulation for VTE treatment not related to cancer [33]. Despite this, the potential of the direct oral anticoagulants for CAT treatment was self-evident. Large, but uncontrolled and single centre series describing the successful use of direct oral anticoagulants for CAT treatment have started to become available [42, 43]. A meta-analysis of the large, direct oral anticoagulant trials considering only included cancer patients $(n=1132)$ indicated that the direct oral anticoagulants were at least as safe and effective as the conventional treatment for VTE in cancer patients [44]. Nevertheless, the main comparator to direct oral anticoagulants in these studies was VKA, clearly not the best therapeutic option for CAT therapy. How would a direct oral anticoagulant perform in CAT when compared to the first-choice agents recommended by guidelines, i.e. LMWH? The answer to the question arrived in 2018 with the Hokusai VTE Cancer (Edoxaban for the Treatment of Cancer-Associated Venous Thromboembolism) trial [45]. In this study, 1046 CAT patients were randomised to receive, after a course of at least 5 days of LMWH, oral edoxaban $60 \mathrm{mg}$ once daily or dalteparin $200 \mathrm{U} \cdot \mathrm{kg}^{-1}$ once daily for 1 month followed by $150 \mathrm{U} \cdot \mathrm{kg}^{-1}$, for at least 6 months and up to 12 months. The primary outcome was a composite of recurrent VTE or major bleeding. Edoxaban was noninferior to dalteparin in CAT patients in a number of primary outcome events ( $12.8 \%$ versus $13.5 \%$; HR $0.97,95 \%$ CI $0.70-1.36 ; \mathrm{p}=0.006$ for noninferiority). When the components of the primary end-point are analysed separately, the results are quite interesting. Contrary to what could be expected, considering results in the non-cancer population, edoxaban patients had a statistical tendency of fewer episodes of VTE recurrence than the dalteparin patients (7.9\% versus $11.3 \%$; 95\% CI -7-0.2), but statistically significantly more major bleeding events (6.9\% versus $4 \%, 95 \%$ CI $0.1-5.6)$. A more careful analysis of the bleeding in the Hokusai VTE Cancer trial demonstrated that the excess of major bleeding with edoxaban was confined to patients with gastrointestinal cancer [46]. Thus, it is worth considering that while edoxaban is now an effective and safe therapeutic alternative to LMWH for CAT treatment, its use in gastrointestinal cancer requires careful benefit-risk weighting. 
TABLE 5 Major randomised trials in cancer-associated thrombosis treatment

\begin{tabular}{|c|c|c|c|c|}
\hline Trial & Year & Patients $\mathrm{n}$ & Drugs evaluated & Major findings \\
\hline CANTHANOX [35] & 2002 & 146 & $\begin{array}{l}\text { Enoxaparin versus } \\
\text { warfarin }\end{array}$ & $\begin{array}{l}\text { Statistical tendency of warfarin to be more associated with major } \\
\text { bleeding }(p=0.09) \text { and death }(p=0.07) \text { than enoxaparin. }\end{array}$ \\
\hline САТCH [37] & 2015 & 900 & $\begin{array}{l}\text { Tinzaparin versus } \\
\text { warfarin }\end{array}$ & $\begin{array}{l}\text { Statistical tendency of VTE recurrence reduction with tinzaparin } \\
(p=07) \text {. No difference in major bleeding or mortality. }\end{array}$ \\
\hline SELECT-D [47] & 2018 & 406 & $\begin{array}{c}\text { Rivaroxaban versus } \\
\text { dalteparin }\end{array}$ & $\begin{array}{c}\text { Statistically significant less VTE recurrence (HR } 0.43,95 \% \mathrm{Cl} \\
0.19-0.99 \text { ) and statistical tendency of more bleeding (HR } 1.83,95 \% \\
\mathrm{Cl} 0.68-4.96 \text { ) with rivaroxaban loesophagus and gastro-oesophageal } \\
\text { junction cancer patients excluded after inclusion of the first } 220 \\
\text { patients) }\end{array}$ \\
\hline
\end{tabular}

VTE: venous thromboembolism.

Another randomised controlled study evaluating a direct oral anticoagulant for CAT treatment was recently published [47]. In the SELECT-D (Comparison of an Oral Factor Xa Inhibitor With Low Molecular Weight Heparin in Patients With Cancer With Venous Thromboembolism) trial, 406 CAT patients were randomised to receive rivaroxaban $15 \mathrm{mg}$ twice daily for 3 weeks followed by $20 \mathrm{mg}$ once daily for a total of 6 months or dalteparin $200 \mathrm{U} \cdot \mathrm{kg}^{-1}$ once daily for 1 month followed by $150 \mathrm{U} \cdot \mathrm{kg}^{-1}$ once daily for 2-6 months. VTE recurrence rates were less frequent with rivaroxaban than dalteparin (4\% versus 11\%; HR 0.43, 95\% CI 0.19-0.99), but similar results to the Hokusai VTE Cancer trial, with a different Xa inhibitor. Bleeding results in SELECT-D are also in line with those from the Hokusai VTE Cancer trial; $6 \%$ of major bleeding was $6 \%$ and $4 \%$ with rivaroxaban and dalteparin, respectively (HR 1.83, 95\% CI 0.68-4.96). Again, gastric/oesophageal cancer patients were especially at high risk for major bleeding and were excluded from a certain point of the study. Apparently, distinct Xa inhibitors have similar profiles of efficacy and safety for the CAT population. Further conclusions in this regard will potentially come with the results of the Caravaggio trial, evaluating apixaban against dalteparin in CAT patients, which is expected in 2019 [48]. Nevertheless, data from the Hokusai VTE Cancer and SELECT-D trials were sufficient to modify the guideline recommendations for CAT treatment. The International Society of Thrombosis and Haemostasis (ISTH) recently released a guidance document, suggesting the use of specific direct oral anticoagulants (rivaroxaban and edoxaban) for CAT treatment in patients with low risk of bleeding and no drug-drug interactions [49]. LMWH are considered an acceptable alternative and are suggested for cancer patients with a higher risk of bleeding with direct oral anticoagulants, such as gastrointestinal cancers. Patients preferences should also be accounted for in the decision-making process. The major randomised trials concerning CAT anticoagulant therapy are summarised in table 5.

\section{Special issues in CAT}

Screening of occult cancer in VTE

VTE can be the first manifestation of cancer and may precede the oncological diagnosis by up to 6 years [50]. However, the risk of cancer in the VTE population is higher during the first 6 months following the acute event [51]. The incidence of occult cancer detection can reach $10 \%$ after the first unprovoked VTE event [52]. In this setting, in an attempt to diagnosis earlier with potential impact on survival, it is logical to pursue a screening strategy of occult cancer in VTE, mainly in the unprovoked patients. However, occult cancer screening has an economic cost, potential radiation exposure and some psychological burden. To answer the question if cancer screening should be performed after an unprovoked VTE event, three large trials were recently published. In the Canadian SOME (Screening for Occult Malignancy in Patients with Idiopathic Venous Thromboembolism) multicentre clinical trial [53], 854 unprovoked VTE patients were randomised to a conservative screening strategy (basic blood testing, chest radiography, and screening for breast, cervical and prostate cancer) or a screening strategy that also included computed tomography (CT) of the abdomen and pelvis. The prevalence of occult cancer was low among both groups after 1 year of follow-up (3.2\% in the limited-screening group versus $4.5 \%$ in the CT group, $\mathrm{p}=0.28$ ), without a significant benefit of routine screening with CT. It is worth noticing that, in SOME, the 
incidence of occult cancer in the unprovoked VTE patients that had a negative screening result and the limited strategy was very low $(0.93 \%$ over the following year), lower than expected and similar to the incidence reported in patients without VTE [54]. In the same direction, an Italian trial of 195 unprovoked VTE patients was randomised to an extensive CT screening strategy (thoracic, abdominal and pelvic) plus a faecal occult blood test or a no standardised testing approach based on the attending physicians' clinical judgment and patients' preferences [31]. No difference was identified in the occult cancer detection efficiency $(10.2 \%$ versus $8.2 \%$, respectively, $\mathrm{p}=0.81$ ). Finally, the additional value of adding positron emission tomography (PET) to the conventional CT detection strategy has also been tested in a French multicentre trial (MVTEP), but again it did not add to the conventional cancer detection strategy [32]. However, the incidence of subsequent diagnosis of cancer during follow-up was significantly lower in patients who had negative PET-CT screening than in patients who had a negative initial limited screening ( $0.5 \%$ versus $4.7 \%$, absolute risk difference $4.1 \%, 95 \%$ CI $0.8-8.4, \mathrm{p}=0.01$ ). Even so, when addressing cost-effectiveness, extensive occult cancer screening failed to prove its usefulness [55]. This led to the actual recommendation of the ISTH, that unprovoked VTE patients should undergo limited cancer screening, including medical evaluation, chest radiograph and laboratory tests: blood count, calcium, urinalysis, and liver function test. Age-specific and sex-specific cancer screening (colon, breast, cervix and prostate) should be performed according to national recommendations [56].

Some particular characteristics of the VTE patients may be suggestive of a more intense pro-coagulant state and, therefore, indicate a higher risk of occult cancer. These patients may eventually benefit from more extensive screening. Among these characteristics are recurrent VTE despite adequate anticoagulant treatment, bilateral deep vein thrombosis and extensive pulmonary embolism. A recent study evaluated the data from the MVTEP trial and identified the following as risk factors for occult cancer in unprovoked VTE patients: age $>50$ years, male sex, high leukocyte counts and high platelet count [57]. Surprisingly, smoker status and history of previous VTE were not associated with occult cancer diagnosis. Age, in particular, has also been identified in a recent meta-analysis as a significant risk factor for occult cancer in unprovoked VTE. Cancer prevalence increased linearly with age and was seven-fold higher in patients aged $\geqslant 50$ years than in younger patients (OR 7.1 (95\% CI 3.1-16)) [58].

Recently, a risk score identifying VTE patients at high risk for occult cancer has been developed. Components of this score include age $>70$ years, chronic lung disease, anaemia, elevated platelet count, prior VTE and recent surgery. Despite being promising, this score, based on data from the Registro Informatizado Enfermedad TromboEmbólica (RIETE), still needs external validation before clinical application [59].

\section{Incidental pulmonary embolism}

The cancer patient is frequently submitted for chest CT scans for several reasons; from the evaluation of disease progression to potential side-effects of chemotherapy in the lung. Incidental pulmonary embolism is a frequent finding on these CT scans, being found in $\sim 3.6 \%$ of patients [60]. Cancer confers to the patient a higher risk of VTE, and asymptomatic pulmonary embolism may be an indicator of an acquired procoagulant state. However, as described previously, cancer patients are more susceptible to both VTE recurrence and major bleeding if anticoagulation is instituted. Thus, what should be the priority? Does asymptomatic pulmonary embolism carry smaller risk than the symptomatic one, specifically in cancer patients? Is this risk low enough to avoid anticoagulation?

Unfortunately, no large uncontrolled trials are available to answer these questions. But smaller studies can provide some guidance on this matter. In a Japanese study, suspected and unsuspected pulmonary embolism in 77 patients with lung cancer did not differ in terms of recurrence $(20 \%$ versus $19 \%, \mathrm{p}=1.0)$ or survival (5.6 versus 6.2 months, $\mathrm{p}=0.85$ ) [61]. Contrary to expected, proximal pulmonary embolism did not result in higher mortality risk than distal pulmonary embolism. Therefore, in cancer patients even small, asymptomatic clots increase the risk of death.

And what about anticoagulation? A Korean study retrospectively evaluated 8014 lung cancer patients [62]. Pulmonary embolism developed in 180 (2.2\%) patients, 113 (63\%) of them were identified incidentally. Of the unsuspected pulmonary embolism patients, 62 (55\%) did not receive anticoagulation therapy and had worse survival than the those receiving anticoagulation therapy (HR 4.1, 95\% CI 2.3-7.6). Again, despite limited evidence and a higher risk of bleeding, anticoagulation appears to be beneficial in incidental CAT. This led to the actual guidelines of the American Society of Clinical Oncology, that recommend that incidental VTE should be treated in the same manner as symptomatic VTE [63].

\section{Duration of anticoagulation for CAT treatment}

Duration of anticoagulation after a CAT episode is still a matter of debate. The majority of randomised trials evaluated anticoagulants drugs for at least 6 months after the acute thrombotic event in cancer $[36,37,45,47]$ and this is the minimum duration of anticoagulation for CAT recommended in most 
guidelines [63-65]. It is also suggested to extend anticoagulation after a CAT episode while the risk factor for VTE recurrence is still present after the initial 6 months, such as active cancer, metastatic disease or chemotherapy use. However, as previously mentioned, CAT patients are at higher risk of bleeding during anticoagulant treatment [34], so the decision of extending anticoagulants beyond 6 months should be considered while balancing the risk of bleeding. However, recent advances in oncologic treatment now provide complete remission of cancer in several patients. In this setting, if CAT has developed and has been adequately treated for 6 months, should anticoagulant therapy be extended? Is it safe to withdraw the anticoagulants? And if so after how long?

One study suggested an elegant way of evaluating the risk of VTE recurrence after the initial 6 months of anticoagulant therapy in CAT patients. In the Cancer-DACUS (Optimal Duration of Low Molecular Weight Heparin for the treatment of Cancer-Related Deep Vein Thrombosis) trial [66], 347 cancer patients who received at least 6 months of LMWH after the first episode of deep vein thrombosis were evaluated with a compression ultrasonography of the legs, and were identified as having residual vein thrombosis $(69.7 \%)$ or not. Residual vein thrombosis patients were then randomised to receive a further 6 months of nadroparin 97 UI per kg twice daily or to stop LMWH. Residual vein thrombosis patients that received 12 months of LMWH did not present lower recurrence rates than the residual vein thrombosis patients who stopped after the first 6 months $(22.7 \%$ versus $21.9 \%$; HR 1.37 , 95\% CI $0.7-2.5$; $\mathrm{p}=0.311)$. However, patients that did not present residual vein thrombosis had a significant lower VTE recurrence than both groups $(2.8 \%$; HR $6,95 \%$ CI $1.7-21.2 ; \mathrm{p}=0.005)$. No difference in major bleeding was identified among the three groups. Therefore, in this study, the absence of residual vein thrombosis after 6 months of CAT treatment identified a population at low risk for recurrent thrombotic events. Residual vein thrombosis absence may identify potential targets of withdrawal of anticoagulant therapy after the initial 6 months of CAT treatment.

\section{Conclusion}

CAT presents peculiar features that distinguished them from ordinary VTE cases. The pathophysiology of CAT includes the production of tissue factor and other procoagulant substances that impair the endothelial balance between thrombosis/anticoagulation. Some cancers are particularly prone to VTE, such as haematological, lung, pancreas, brain and stomach. Other properties are related to VTE risks, such as chemotherapy use and advanced staging. CAT carry a high risk of VTE recurrence but also major bleeding, when treated. Treatment of CAT requires a different approach than ordinary VTE cases, and LMWH is the first treatment choice. However, direct oral anticoagulants (edoxaban and rivaroxaban) proved to be as efficient and safe as LMWH for CAT treatment, and are a viable choice, with potential benefits in adherence. CAT may present other clinical challenges such as the lack of evidence supporting extensive cancer screening in unprovoked VTE, the need for treatment even in incidental VTE in cancer patients, and the absence of consensus regarding primary VTE prophylaxis in high-risk ambulatory cancer patients. In cancer patients admitted for major surgery, primary prophylaxis with LMWH for 30 days impacted positively in VTE recurrence and should be implemented. Still, upcoming data from large cohorts are expected for the near future potentially bringing enlightenment to an intriguing scenario in which multiple factors significantly influence the clinical course of VTE, turning CAT into a specific clinical condition.

Conflict of interest: C.J. Fernandes reports personal fees from Actelion, GSK and Bayer, outside the submitted work. L.T.K. Morinaga reports personal fees from Bayer, outside the submitted work. J.L. Alves Jr reports personal fees from Bayer and Actelion, outside the submitted work. M.A. Castro has nothing to disclose. D. Calderaro reports personal fees from Pfizer and Bayer, outside the submitted work. C.V.P. Jardim reports personal fees from Bayer and Actelion, and other funding from GSK (former employee), outside the submitted work. R. Souza reports personal fees from Actelion, GSK, Pfizer and Bayer, outside the submitted work.

Support statement: This study was funded by the Fundação de Amparo à Pesquisa do Estado de São Paulo (\#2017/ 26871-9). Funding information for this article has been deposited with the Crossref Funder Registry.

\section{References}

1 ISTH Steering Committee for World Thrombosis Day. Thrombosis: a major contributor to global disease burden. Thromb Haemost 2014; 112: 843-852.

2 Fernandes CJCDS, Jardim CVP, Alves JL Jr, et al. Reperfusion in acute pulmonary thromboembolism. $J$ Bras Pneumol 2018; 44: 237-243.

3 Elyamany G, Alzahrani AM, Bukhary E. Cancer-associated thrombosis: an overview. Clin Med Insights Oncol 2014; 8: 129-137.

4 Heit JA, Spencer FA, White RH. The epidemiology of venous thromboembolism. J Thromb Thrombolysis 2016; 41: 3-14.

5 Laporte S, Mismetti P, Decousus H, et al. Clinical predictors for fatal pulmonary embolism in 15,520 patients with venous thromboembolism: findings from the Registro Informatizado de la Enfermedad TromboEmbolica venosa (RIETE) Registry. Circulation 2008; 117: 1711-1716. 
6 Puurunen MK, Gona PN, Larson MG, et al. Epidemiology of venous thromboembolism in the Framingham Heart Study. Thromb Res 2016; 145: 27-33.

7 Turpie AGG, Haas S, Weitz JI, et al. GARFIELD-VTE: 6-month outcomes. Res Pract Thromb Haemost 2017; 1: $1-15$.

8 Khorana AA, Francis CW, Culakova E, et al. Thromboembolism is a leading cause of death in cancer patients receiving outpatient chemotherapy. J Thromb Haemost 2007; 5: 632-634.

9 Mukai M, Oka T. Mechanism and management of cancer-associated thrombosis. J Cardiol 2018; 72: 89-93.

10 Blann AD, Lip GY. Venous thromboembolism. BMJ 2006; 332: 215-219.

11 Blann AD, Dunmore S. Arterial and venous thrombosis in cancer patients. Cardiol Res Pract 2011; 2011: 394740.

12 Ades S, Kumar S, Alam M, et al. Tumor oncogene (KRAS) status and risk of venous thrombosis in patients with metastatic colorectal cancer. J Thromb Haemost 2015; 13: 998-1003.

13 Corrales-Rodriguez L, Soulieres D, Weng X, et al. Mutations in NSCLC and their link with lung cancer-associated thrombosis: a case-control study. Thromb Res 2014; 133: 48-51.

14 Nielsen C, Birgens HS, Nordestgaard BG, et al. The JAK2V617F somatic mutation, mortality and cancer risk in the general population. Haematologica 2011; 96: 450-453.

15 Shetty S, Kulkarni B, Pai N, et al. JAK2 mutations across a spectrum of venous thrombosis cases. Am J Clin Pathol 2010; 134: 82-85.

16 Stein PD, Beemath A, Meyers FA, et al. Incidence of venous thromboembolism in patients hospitalized with cancer. Am J Med 2006; 119: 60-68.

17 Fernandes C. Evolution in the management of non-small cell lung cancer in Brazil. J Bras Pneumol 2017; 43: 403-404.

18 Gade IL, Braekkan SK, Naess IA, et al. The impact of initial cancer stage on the incidence of venous thromboembolism: the Scandinavian Thrombosis and Cancer (STAC) Cohort. J Thromb Haemost 2017; 15: 1567-1575.

19 Blom JW, Doggen CJ, Osanto S, et al. Malignancies, prothrombotic mutations, and the risk of venous thrombosis. JAMA 2005; 293: 715-722.

20 Trujillo-Santos J, Nieto JA, Tiberio G, et al. Predicting recurrences or major bleeding in cancer patients with venous thromboembolism. Findings from the RIETE Registry. Thromb Haemost 2008; 100: 435-439.

21 Walker AJ, Card TR, West J, et al. Incidence of venous thromboembolism in patients with cancer - a cohort study using linked United Kingdom databases. Eur J Cancer 2013; 49: 1404-1413.

22 Falanga A, Russo L. Epidemiology, risk and outcomes of venous thromboembolism in cancer. Hamostaseologie 2012; 32: 115-125

23 Imberti D, Benedetti R. Primary prophylaxis of VTE in cancer outpatients. Thromb Res 2016; 140: Suppl 1, S103-S108.

24 Khorana AA, Kuderer NM, Culakova E, et al. Development and validation of a predictive model for chemotherapy-associated thrombosis. Blood 2008; 111: 4902-4907.

25 Ay C, Dunkler D, Marosi C, et al. Prediction of venous thromboembolism in cancer patients. Blood 2010; 116: 5377-5382.

26 Khorana AA, McCrae KR. Risk stratification strategies for cancer-associated thrombosis: an update. Thromb Res 2014; 133: Suppl 2, S35-S38.

27 Agnelli G, Gussoni G, Bianchini C, et al. Nadroparin for the prevention of thromboembolic events in ambulatory patients with metastatic or locally advanced solid cancer receiving chemotherapy: a randomised, placebo-controlled, double-blind study. Lancet Oncol 2009; 10: 943-949.

28 Agnelli G, George DJ, Kakkar AK, et al. Semuloparin for thromboprophylaxis in patients receiving chemotherapy for cancer. N Engl J Med 2012; 366: 601-609.

29 Di Nisio M, Porreca E, Candeloro M, et al. Primary prophylaxis for venous thromboembolism in ambulatory cancer patients receiving chemotherapy. Cochrane Database Syst Rev 2016; 12: CD008500.

30 Carrier M, Abou-Nassar K, Mallick R, et al. Apixaban to prevent venous thromboembolism in patients with cancer. N Engl J Med 2019; 380: 711-719.

31 Prandoni P, Bernardi E, Valle FD, et al. Extensive computed tomography versus limited screening for detection of occult cancer in unprovoked venous thromboembolism: a multicenter, controlled, randomized clinical trial. Semin Thromb Hemost 2016; 42: 884-890.

32 Robin P, Le Roux PY, Planquette B, et al. Limited screening with versus without (18)F-fluorodeoxyglucose PET/ CT for occult malignancy in unprovoked venous thromboembolism: an open-label randomised controlled trial. Lancet Oncol 2016; 17: 193-199.

33 Kearon C, Akl EA, Ornelas J, et al. Antithrombotic therapy for VTE disease: CHEST Guideline and Expert Panel Report. Chest 2016; 149: 315-352.

34 Prandoni P, Lensing AW, Piccioli A, et al. Recurrent venous thromboembolism and bleeding complications during anticoagulant treatment in patients with cancer and venous thrombosis. Blood 2002; 100: 3484-3488.

35 Meyer G, Marjanovic Z, Valcke J, et al. Comparison of low-molecular-weight heparin and warfarin for the secondary prevention of venous thromboembolism in patients with cancer: a randomized controlled study. Arch Intern Med 2002; 162: 1729-1735.

36 Lee AY, Levine MN, Baker RI, et al. Low-molecular-weight heparin versus a coumarin for the prevention of recurrent venous thromboembolism in patients with cancer. N Engl J Med 2003; 349: 146-153.

37 Lee AYY, Kamphuisen PW, Meyer G, et al. Tinzaparin vs warfarin for treatment of acute venous thromboembolism in patients with active cancer: a randomized clinical trial. JAMA 2015; 314: 677-686.

38 Hakoum MB, Kahale LA, Tsolakian IG, et al. Anticoagulation for the initial treatment of venous thromboembolism in people with cancer. Cochrane Database Syst Rev 2018; 1: CD006649.

39 Akl EA, Kahale L, Barba M, et al. Anticoagulation for the long-term treatment of venous thromboembolism in patients with cancer. Cochrane Database Syst Rev 2014; 7: CD006650.

40 Francis CW, Kessler CM, Goldhaber SZ, et al. Treatment of venous thromboembolism in cancer patients with dalteparin for up to 12 months: the DALTECAN Study. J Thromb Haemost 2015; 13: 1028-1035.

41 Fernandes CJ, Alves Junior JL, Gavilanes F, et al. New anticoagulants for the treatment of venous thromboembolism. J Bras Pneumol 2016; 42: 146-154. 
42 Xavier FD, Hoff PMG, Braghiroli MI, et al. Rivaroxaban: an affordable and effective alternative in cancer-related thrombosis? J Glob Oncol 2017; 3: 15-22.

43 Pignataro BS, Nishinari K, Cavalcante RN, et al. Oral rivaroxaban for the treatment of symptomatic venous thromboembolism in 400 patients with active cancer: a single-center experience. Clin Appl Thromb Hemost 2017; 23: 883-887.

44 Vedovati MC, Germini F, Agnelli G, et al. Direct oral anticoagulants in patients with VTE and cancer: a systematic review and meta-analysis. Chest 2015; 147: 475-483.

45 Raskob GE, van Es N, Verhamme $\mathrm{P}$, et al. Edoxaban for the treatment of cancer-associated venous thromboembolism. N Engl J Med 2018; 378: 615-624.

46 Kraaijpoel N, Di Nisio M, Mulder FI, et al. Clinical impact of bleeding in cancer-associated venous thromboembolism: results from the Hokusai VTE Cancer Study. Thromb Haemost 2018; 118: 1439-1449.

47 Young AM, Marshall A, Thirlwall J, et al. Comparison of an oral factor Xa inhibitor with low molecular weight heparin in patients with cancer with venous thromboembolism: results of a randomized trial (SELECT-D). J Clin Oncol 2018; 36: 2017-2023.

48 Agnelli G, Becattini C, Bauersachs R, et al. Apixaban versus dalteparin for the treatment of acute venous thromboembolism in patients with cancer: the Caravaggio Study. Thromb Haemost 2018; 118: 1668-1678.

49 Khorana AA, Noble S, Lee AYY, et al. Role of direct oral anticoagulants in the treatment of cancer-associated venous thromboembolism: guidance from the SSC of the ISTH. J Thromb Haemost 2018; 16: 1891-1894.

50 Saba HI, Khalil FK, Morelli GA, et al. Thromboembolism preceding cancer: a correlation study. Am J Hematol 2003; 72: 109-114

51 Schulman S, Lindmarker P. Incidence of cancer after prophylaxis with warfarin against recurrent venous thromboembolism. Duration of Anticoagulation Trial. N Engl J Med 2000; 342: 1953-1958.

52 Carrier M, Le Gal G, Wells PS, et al. Systematic review: the Trousseau syndrome revisited: should we screen extensively for cancer in patients with venous thromboembolism? Ann Intern Med 2008; 149: 323-333.

53 Carrier M, Lazo-Langner A, Shivakumar S, et al. Screening for occult cancer in unprovoked venous thromboembolism. N Engl J Med 2015; 373: 697-704.

54 Prandoni P, Casiglia E, Piccioli A, et al. The risk of cancer in patients with venous thromboembolism does not exceed that expected in the general population after the first 6 months. J Thromb Haemost 2010; 8: 1126-1127.

55 Coyle K, Carrier M, Lazo-Langner A, et al. Cost effectiveness of the addition of a comprehensive CT scan to the abdomen and pelvis for the detection of cancer after unprovoked venous thromboembolism. Thromb Res 2017; 151: 67-71.

56 Delluc A, Antic D, Lecumberri R, et al. Occult cancer screening in patients with venous thromboembolism: guidance from the SSC of the ISTH. J Thromb Haemost 2017; 15: 2076-2079.

57 Robin P, Le Roux PY, Tromeur C, et al. Risk factors of occult malignancy in patients with unprovoked venous thromboembolism. Thromb Res 2017; 159: 48-51.

58 van Es N, Le Gal G, Otten HM, et al. Screening for occult cancer in patients with unprovoked venous thromboembolism: a systematic review and meta-analysis of individual patient data. Ann Intern Med 2017; 167: 410-417.

59 Jara-Palomares L, Otero R, Jimenez D, et al. Development of a risk prediction score for occult cancer in patients with VTE. Chest 2017; 151: 564-571.

60 Klok FA, Huisman MV. Management of incidental pulmonary embolism. Eur Respir J 2017; 49: 1700275

61 Shinagare AB, Okajima Y, Oxnard GR, et al. Unsuspected pulmonary embolism in lung cancer patients: comparison of clinical characteristics and outcome with suspected pulmonary embolism. Lung Cancer 2012; 78: 161-166.

62 Sun JM, Kim TS, Lee J, et al. Unsuspected pulmonary emboli in lung cancer patients: the impact on survival and the significance of anticoagulation therapy. Lung Cancer 2010; 69: 330-336.

63 Lyman GH, Bohlke K, Khorana AA, et al. Venous thromboembolism prophylaxis and treatment in patients with cancer: American Society of Clinical Oncology Clinical Practice Guideline Update 2014. J Clin Oncol 2015; 33: 654-656.

64 Watson HG, Keeling DM, Laffan M, et al. Guideline on aspects of cancer-related venous thrombosis. $\mathrm{Br} J$ Haematol 2015; 170: 640-648.

65 Mandala M, Falanga A, Roila F, et al. Management of venous thromboembolism (VTE) in cancer patients: ESMO Clinical Practice Guidelines. Ann Oncol 2011; 22: Suppl 6, vi85-vi92.

66 Napolitano M, Saccullo G, Malato A, et al. Optimal duration of low molecular weight heparin for the treatment of cancer-related deep vein thrombosis: the Cancer-DACUS Study. J Clin Oncol 2014; 32: 3607-3612. 